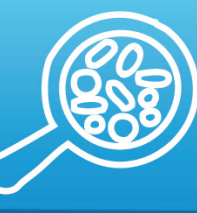

MICROBIOLOGY

\title{
Antifungal effect of Atorvastatin against Candida species in comparison to Fluconazole and Nystatin
}

Ava Nasr Esfahani ${ }^{1}$, Zahra Golestannejad ${ }^{2}$, Faezeh Khozeimeh ${ }^{2}$, Parvin Dehghan ${ }^{3}$, Mehrnoosh Maheronnaghsh ${ }^{3}$, Zahra Zarei $^{4}$

1) Dental Research Center, School of Dentistry, Isfahan University of Medical Sciences, Isfahan, Islamic Republic of Iran

2) Department of Oral Medicine, Dental Research Center, School of Dentistry, Isfahan University of Medical Sciences, Isfahan, Islamic Republic of Iran

3) Department of Mycology and Parasitology, School of Medicine, Isfahan University of Medical Sciences, Isfahan, Islamic Republic of Iran

4) Department of Orthodontics, Dental Research Center, School of Dentistry, Isfahan University of Medical Sciences, Isfahan, Islamic Republic of Iran
DOI: $10.15386 / \mathrm{mpr}-1209$

Manuscript received: 21.10 .2018

Received in revised form: 13.02 .2019

Accepted: 13.03.2019

Address for correspondence:

z.zarei72@yahoo.com

\begin{abstract}
Background and aims. Atorvastatin is a plasma cholesterol-lowering drug which applies antifungal effects by inhibiting the production of yeast cell wall ergostrol. The aim of present study was to investigate in-vitro susceptibility of candida species to atorvastatin, in comparison to nystatin and fluconazole.
\end{abstract}

Methods. Minimum inhibitory concentrations (MIC) and minimum fungicidal concentrations (MFC) were determined using serial dilution. Candida strains isolated from 35 patients receiving cancer chemotherapy in Isfahan, Seyyed-al-Shohada Hospital and analyzed by Kruskal-Wallis and Mann Whitney statistical methods.

Results. Candida isolates included 5 strains, C. albicans, C. glabrata, C. kefyr, C. stellatoidea and $C$. krusei. All five strains appeared to be resistant to nystatin and fluconazole but sensitive to atorvastatin with no statistically significant difference. The MFC of atorvastatin was significantly lower in comparison to both nystatin and fluconazole for all five strains ( $\mathrm{p}$ value $<0.05$ ). There was no significant difference between the MFCs of 5 strains for fluconazole and atorvastatin. However, MFC of nystatin differed significantly for $C$. albicans and $C$. kefyr $(\mathrm{p}=0.007)$.

Conclusion. The results showed that all strains were sensitive to atorvastatin and resistant to nystatin and fluconazole. Atorvastatin MIC for C. albicans, C. krusei and C. stellatoidea was equivalent to its serum level used to treat hyperlipidemia and was above such level for both C. glabrata and C. kefyr.

Keywords: oral candidiasis, fluconazole, Nystatin, Atorvastatin, cancer chemotherapy

\section{Introduction}

Treatment of cancer using chemotherapy improves disease prognosis but is associated with several side effects including oral candidiasis, which is a major source of illness in these patients [1].

Since the 1990s changes have been observed in species that cause candidiasis in patients with malignancy. In these patients, a distinct shift of Candida albicans strains to non-albicans is noticed [2-4].

A systematic study by Cochrane in 2010 evaluated the effectiveness of antifungal drugs on candida infection in cancer patients receiving chemotherapy and revealed that there was insufficient evidence for the usefulness of existing antifungal agents, including fluconazole and nystatin, for treating oral candidiasis in these patients [1]. Several reports have demonstrated that $C$. glabrata and C. krusei are inherently resistant to fluconazole $[5,6]$.

Furthermore, prophylactic use of antifungal agents has developed acquired resistance in such patients $[7,8]$.

Therefore, searching for new antifungal agents for patients undergoing chemotherapy appears to be of great importance.

Recently, antifungal activity of statins has been investigated. The hypothesis behind was the decrease in the number of reported cases of zygomycosis in the patients with diabetes mellitus, due to the widespread use of statins [9].

Statins lower serum cholesterol levels through inhibition of HMG-CoA (3-hydroxy-3-methylglutaryl-CoA) reductase, an essential enzyme for cholesterol biosynthesis. Yeasts also use 
this enzymatic pathway, but their end-product is ergosterol rather than cholesterol [10-12]. Also, statins deregulate production of isoprenoid which leads to cellular respiratory deficiency, mitochondrial dysfunction and lipid change in plasma membrane of C. albicans [12-14].

Several studies have shown the potential antifungal activity of the statins on different pathogenic fungi $[12,15$ 22]. Zhou et al. in 2018 demonstrated synergistic activities of Lovastatin and Itraconazole combination especially in C. albicans biofilms as well as its potential clinical administration particularly against the azole drug-resistant mutants [23]. Ting et al. in a systematic review concluded that statins have antifungal properties against Candida albicans, Aspergillus fumigatus, and Zygomycetes spp [24]. However, no data have been found about the antifungal effect of statins against candida strains in patients with cancer receiving chemotherapy.

The aim of present study was to determine the species causing oral candidiasis in patients with cancer receiving chemotherapy and also to investigate the in vitro susceptibility of these species to one of the most commercially available statins, atorvastatin, in comparison with nystatin and fluconazole.

\section{Materials and methods}

\section{Collection and isolation of strains}

The candida strains used in this study were isolated from 35 patients with oral candidiasis under chemotherapy hospitalized in Isfahan $\left(32.6546^{\circ} \mathrm{N}, 51.6680^{\circ} \mathrm{E}\right)$, Seyyed al-Shohada Hospital. The isolates originated from culture collection of the Isfahan Medical School of Mycology were identified by CHROMagar candida medium (CHROMagar Company, Paris, France) and PCR-RFLP molecular method [25].

The obtained fungal strains were activated, out of which, 28 samples were successfully turned up and the other samples were excluded from the study. Finally, 28 active samples were identified: 19 C. albicans, 4 C. glabrata, 3 C. kefyr strains, 1 C. stelatoidea and 1 C. krusei. 24 hours prior to testing, the isolates were passaged twice on Sabouraud dextrose agar (SDA) to confirm the purity of the isolates.

For each isolate, inoculum suspension was adjusted to a $0.5 \mathrm{McF}$ arland standard. The optical absorption of the suspension at $530 \mathrm{~nm}$ was measured by WPA Biowave II spectrophotometer (Biochrom, Cambridge, UK).

\section{Antifungal agents}

Nystatin (Sigma-Aldrich, Taufkirchen, Germany), fluconazole (Sigma-Aldrich, Taufkirchen, Germany) and atorvastatin (Sigma-Aldrich, Taufkirchen, Germany) were tested against all the strains. To prepare stock solutions of each drug, fluconazole and atorvastatin were diluted with dimethyl sulfoxide (DMSO) (Merck, Darmstadt, Hesse, Germany), and Nystatin was dissolved in methanol. Stock solutions were stored at $-20^{\circ} \mathrm{C}$.

\section{Antifungal susceptibility testing}

The in vitro antimycotic effect of atorvastatin, fluconazole and nystatin was determined using the broth microdilution method according to the M27-A3 guideline of the Clinical and laboratory standards institute (CLSI) [26]. The minimal inhibitory concentration (MIC) values were determined in 96-well microplates by visual assessment.

The final concentration range was $0.25-128 \mu \mathrm{g} / \mathrm{ml}$ for fluconazole and $0.5-256 \mu \mathrm{g} / \mathrm{ml}$ for atorvastatin and nystatin. $100 \mu \mathrm{l}$ of fungal suspension was added to microplates. The positive control wells contained $100 \mu$ of yeast suspension and $100 \mu \mathrm{l}$ of RPMI 1640 (Sigma-Aldrich, Taufkirchen, Germany). For negative control $200 \mu$ of RPMI 1640 was added. The microplates were incubated at $35^{\circ} \mathrm{C}$ for $24-48 \mathrm{~h}$. The MICs were determined as the lowest drug concentration that produced an optically clear well. All experiments repeated three times.

\section{Minimum fungicidal concentration assay}

In order to determine the minimum fungicidal concentration (MFC), $21 \mu \mathrm{L}$ of MIC well and subsequent wells were subcultured onto SDA. The MFC was determined as the lowest drug concentration that fewer than three fungal colonies were observed after 24-48 $\mathrm{h}$ incubation at $35^{\circ} \mathrm{C}$. The MFC determinations repeated three times.

\section{Statistical analysis}

Data analysis was conducted with SPSS software [22]. For each strain mode, median and range were calculated. Kruskal-wallis test was performed to compare the MIC and MFC levels of the three tested drugs (nystatin, fluconazole and atorvastatin) with each other against five different strains used in this study. If there was a significant difference ( $\mathrm{p}$ value $<0.05$ ) the Mann-Whitney test was performed to compare the antifungal effects of drugs (MIC and MFC) in binary groups with each other. The significance level in this analysis was 0.05 .

\section{Results}

The sample consisted of candida strains isolated from patients with oral candidiasis under chemotherapy hospitalized in Isfahan, Seyyed-alShohada hospital. PCRRFLP molecular method determined the strains as followed: 19 C. albicans, 4 C. glabrata, 3 C. kefyr, 1 C. stellatoidea, 1 C. krusei.

Median, mode and range of The minimum inhibitory concentration (MIC) of atorvastatin, nystatin and fluconazole against five types of candida strains isolated from the mouth of patients undergoing chemotherapy is shown in Table I.

For each of the three drugs, nystatin, fluconazole and atorvastatin, there was no significant difference between the MICs for five strains ( $p$-value was calculated $0.73,1.000$ and 0.89 respectively). 
Table I. Median, mode and range of the minimum inhibitory concentration (MIC) of atorvastatin, nystatin and fluconazole against five types of candida strains.

\begin{tabular}{|c|c|c|c|c|}
\hline Strain & MIC & Nystatin & Fluconazole & Atorvastatin \\
\hline \multirow{3}{*}{ C. albicans [19] } & Range & $128->256$ & $>256$ & $1-256$ \\
\hline & Median & $>256$ & $>256$ & 8 \\
\hline & Mode & $>256$ & $>256$ & 4 \\
\hline \multirow{3}{*}{ C. glabrata [4] } & Range & $\geq 256$ & $>256$ & $4-8$ \\
\hline & Median & $>256$ & $>256$ & 4 \\
\hline & Mode & $>256$ & $>256$ & 4 \\
\hline \multirow{3}{*}{ C. kefyr [3] } & Range & $\geq 256$ & $>256$ & $4-16$ \\
\hline & Median & $>256$ & $>256$ & 8 \\
\hline & Mode & $>256$ & $>256$ & 8 \\
\hline \multirow{3}{*}{ C. stellatoidea [1] } & Range & $>256$ & $>256$ & 16 \\
\hline & Median & $>256$ & $>256$ & 16 \\
\hline & Mode & $>256$ & $>256$ & 16 \\
\hline \multirow{3}{*}{ C. krusei [1] } & Range & 256 & $>256$ & 8 \\
\hline & Median & 256 & $>256$ & 8 \\
\hline & Mode & 256 & $>256$ & 8 \\
\hline
\end{tabular}

P-values obtained from the comparison of the MIC values for the three drugs against each strains are: $C$. albicans $<0.001$, C. glabrata $<0.001$, C. kefyr $=0.005$, C. Stellatoidea $=0.021, C$. krusei $=0.021$. The results of the following MannWhitney analysis are represented In Table II.

Furthermore, considering that for all three medications, the break point is $128 \mu \mathrm{g} / \mathrm{ml}$, if the MIC for each strain was higher than the break point, it would indicate resistance. And if it is lower than the break point, it would indicate the susceptibility of the strain to that drug.
The above results evidenced that all of five tested strains were resistant to fluconazole and nystatin without significant differences between them (median MIC > $128 \mu \mathrm{g} / \mathrm{ml}$ and $\mathrm{p}$-value $>0.05$ ). But all strains showed susceptibility to atorvastatin without significant differences. (median MIC $<128 \mu \mathrm{g} / \mathrm{ml}$ and $\mathrm{p}$-value $<0.05$ ).

Median, mode and range of the MFC of atorvastatin, nystatin and fluconazole on the five types of candida strains isolated from the mouth of patients undergoing chemotherapy are shown in Table III.

Table II. Comparison of MIC values of drugs pairwise against strains by the Mann-Whitney analysis.

\begin{tabular}{l|c|c|c|c|c}
\hline Drugs & C.albicans & C.glabrata & C.kefyr & C.stellatoidea & C.krusei \\
\hline Nystatin-Fluconazole & 0.000 & 0.319 & 0.258 & 1.000 & 0.025 \\
Nystatin-Atorvastatin & 0.000 & 0.000 & 0.000 & 0.025 & 0.025 \\
Fluconazole-Atorvastatin & 0.000 & 0.000 & 0.000 & 0.025 & 0.025
\end{tabular}

Table III. Median, mode and range of the minimum fungicidal concentration (MFC) of atorvastatin, nystatin and fluconazole against five types of candida strains.

\begin{tabular}{|c|c|c|c|c|}
\hline Strain & MFC & Nystatin & Fluconazole & Atorvastatin \\
\hline \multirow{3}{*}{ C. albicans [19] } & Range & $256->256$ & $>256$ & $4->256$ \\
\hline & Median & $>256$ & $>256$ & 32 \\
\hline & Mode & $>256$ & $>256$ & 16 \\
\hline \multirow{3}{*}{ C. glabrata [4] } & Range & $>256$ & $>256$ & $16->256$ \\
\hline & Median & $>256$ & $>256$ & 80 \\
\hline & Mode & $>256$ & $>256$ & 16 \\
\hline \multirow{3}{*}{ C. kefyr [3] } & Range & $256->256$ & $>256$ & $16->256$ \\
\hline & Median & $>256$ & $>256$ & 128 \\
\hline & Mode & $>256$ & $>256$ & 128 \\
\hline \multirow{3}{*}{ C. stellatoidea [1] } & Range & $>256$ & $>256$ & $16-32$ \\
\hline & Median & $>256$ & $>256$ & 32 \\
\hline & Mode & $>256$ & $>256$ & 32 \\
\hline \multirow{3}{*}{ C. krusei [1] } & Range & $>256$ & $>256$ & 8 \\
\hline & Median & $>256$ & $>256$ & 8 \\
\hline & Mode & $>256$ & $>256$ & 8 \\
\hline
\end{tabular}


Table IV. Comparison of MFC values of drugs pairwise against strains by the Mann-Whitney analysis.

\begin{tabular}{l|c|c|c|c|c}
\hline Drugs & C.albicans & C.glabrata & C.kefyr & C.stellatoidea & C.krusei \\
\hline Nystatin-Fluconazole & 0.081 & 1.000 & 0.258 & 1.000 & 1.000 \\
Nystatin-Atorvastatin & 0.000 & 0.001 & 0.050 & 0.034 & 0.034 \\
Fluconazole-Atorvastatin & 0.000 & 0.001 & 0.004 & 0.034 & 0.034
\end{tabular}

According to the results, MFC of atorvastatin was significantly lower than both nystatin and fluconazole in all five strains, which shows higher antifungal effect for atorvastatin, against all five strains, than the other two drugs (Table IV).

There were no significant differences between fluconazole and atorvastatin MFCs against the five strains $(\mathrm{p}=1$ and 0.24 respectively) but it was significantly different in nystatin $(\mathrm{p}=0.028)$. Mann-Whitney analysis results showed that only the MFC obtained for C. albicans and $C$. kefyr had significant difference $(\mathrm{p}=0.007)$. As for the rest of the strains, the MFC concentration showed no significant differences $(\mathrm{p}>0.05)$.

\section{Discussion}

The antifungal effect of atorvastatin against Candida strains isolated from the mouth of patients undergoing chemotherapy was evaluated. The results showed that atorvastatin had more growth inhibitory and fungicidal effect on all Candida strains isolated from these patients compared to both Nystatin and fluconazole.

There are similar reports which demonstrated the resistance of oral candida strains isolated from patients receiving chemotherapy to polyenes [27,28], and fluconazole $[29,30]$ respectively.

Widespread prophylactic use of routine antifungal drugs in such patients has led to acquired resistance of isolated strains [7]. Also, studies have shown inherent resistance of C. krusei and C. glabrata to polyenes and azoles $[5,8]$. Since nystatin, fluconazole and atorvastatin have different mechanisms of action, acquired or inherent resistance of Candida strains to polyenes and azoles does not result in cross-resistance to atorvastatin. Statins interfere with isoprenoid production, thus lead to mitochondrial dysfunction and impaired cellular respiration. They also alter the dynamics of the cell membrane of $C$. albicans by changing the structure and metabolism of lipids [12-14]. While nystatin, by creating multiple pores in the yeast wall, increases the permeability and osmotic imbalance, ultimately resulting in the rupture of the yeast wall [31]. Fluconazole, on the other hand, affects yeasts by disrupting the sterol $14 \alpha$-Demethylase (cyp51) enzyme function, which is one of the essential enzymes in the formation of the yeast wall [31].
Another possible reason for the resistance of candida strains in chemotherapy patients is the change in the physiological conditions, including the quantitative and qualitative changes in saliva following chemotherapy, resulting in the development of resistant antifungal strains [32]. It has been suggested that hyposalivation following head and neck radiotherapy is associated with the emersion of drug-resistant Candida strains [33]. Studies have shown that environmental changes result in rapid alterations in the gene expression of $C$. albicans and its adaptation to the environment [34]. Most of the infection-related changes in the $C$. albicans gene are a reflection of its environmental adaption [35].

Other results of this study demonstrated that, MIC of atorvastatin for three strains out of five ones tested, including krusei, stellatoidea, and albicans is similar to the therapeutic serum level of the drug used in the treatment of hyperlipidemia, which is reported in various studies to be in the range of 10 to $80 \mathrm{mg}$ [36]. In accordance with this part of the study, Brilhante et al. [20] in 2015 showed that, MIC of simvastatin (of statins category) against $C$. albicans is equal to its serum levels used to control hyperlipidemia. On the other hand, in the present study, MIC of atorvastatin against the other two strains (C. glabrata and C. kefyr) was obtained higher than its serum level in hyperlipidemia. Macreadie et al. [12] in 2006, and Qiao et al. [37] in 2007 reported that the MIC of Atorvastatin against Aspergillus fumigatus was much higher than the serum level required for control and treatment of hyperlipidemia, as shown in our study.

In vitro assessment of the antifungal effect of Atorvastatin, Nystatin and Fluconazole, is considered to be the main constraint to the present study, and we recommend conducting subsequent clinical studies (in vivo), including topical and systemic administration of atorvastatin, in the treatment of candida lesions of chemotherapy patients.

Overall, this study showed that all the five strains isolated from chemotherapy patients were susceptible to atorvastatin and resistant to nystatin and fluconazole. However, three strains including krusei, stellatoidea, and albicans had MIC levels equal to the serum levels of atorvastatin used in the treatment of hyperlipidemia and two strains, including C. glabrata and C. kefyr, had higher MIC levels. Therefore, Atorvastatin can be considered as one of the antifungal treatments against some candida strain in chemotherapy patients. 


\section{References}

1. Worthington HV, Clarkson JE, Khalid T, Meyer S, McCabe $\mathrm{M}$. Interventions for treating oral candidiasis for patients with cancer receiving treatment. Cochrane Database Syst Rev. 2010;(7):CD001972.

2. Antoniadou A, Torres HA, Lewis RE, Thornby J, Bodey GP, Tarrand JP, et al. Candidemia in a tertiary care cancer center: in vitro susceptibility and its association with outcome of initial antifungal therapy. Medicine (Baltimore). 2003;82:309-321.

3. Hachem R, Hanna H, Kontoyiannis D, Jiang Y, Raad I. The changing epidemiology of invasive candidiasis: Candida glabrata and Candida krusei as the leading causes of candidemia in hematologic malignancy. Cancer. 2008;112:2493-2499.

4. Sipsas NV, Lewis RE, Tarrand J, Hachem R, Rolston KV, Raad II, et al. Candidemia in patients with hematologic malignancies in the era of new antifungal agents (2001-2007): stable incidence but changing epidemiology of a still frequently lethal infection. Cancer. 2009;115:4745-4752.

5. Krcmery V, Barnes AJ. Non-albicans Candida spp. causing fungaemia: pathogenicity and antifungal resistance. J Hosp Infect. 2002;50:243-260.

6. Sanglard D, Odds FC. Resistance of Candida species to antifungal agents: molecular mechanisms and clinical consequences. Lancet Infect Dis. 2002;2:73-85.

7. Bennett JE, Izumikawa K, Marr KA. Mechanism of increased fluconazole resistance in Candida glabrata during prophylaxis. Antimicrob Agents Chemother. 2004;48:1773-1777.

8. Rex JH, Rinaldi MG, Pfaller MA. Resistance of Candida species to fluconazole. Antimicrob Agents Chemother. 1995;39:1-8.

9. Kontoyiannis DP. Decrease in the number of reported cases of zygomycosis among patients with diabetes mellitus: a hypothesis. Clin Infect Dis. 2007;44:1089-1090.

10. Wikhe K, Westermeyer C, Macreadie IG. Biological consequences of statins in Candida species and possible implications for human health. Biochem Soc Trans. 2007;35:1529-1532.

11. Forrest GN, Kopack AM, Perencevich EN. Statins in candidemia: clinical outcomes from a matched cohort study. BMC Infect Dis. 2010;10:152.

12. Macreadie IG, Johnson G, Schlosser T, Macreadie PI. Growth inhibition of Candida species and Aspergillus fumigatus by statins. FEMS Microbiol Lett. 2006;262:9-13.

13. Westermeyer C, Macreadie IG. Simvastatin reduces ergosterol levels, inhibits growth and causes loss of mtDNA in Candida glabrata. FEMS Yeast Res. 2007;7:436-441.

14. Gyetvai A, Emri T, Takács K, Dergez T, Fekete A, Pesti M, et al. Lovastatin possesses a fungistatic effect against Candida albicans, but does not trigger apoptosis in this opportunistic human pathogen. FEMS Yeast Res. 2006;6:1140-1148.

15. Nyilasi I, Kocsubé S, Pesti M, Lukács G, Papp T, Vágvölgyi C. In vitro interactions between primycin and different statins in their effects against some clinically important fungi. J Med Microbiol. 2010;59:200-205.
16. Chamilos G, Lewis RE, Kontoyiannis DP. Lovastatin has significant activity against zygomycetes and interacts synergistically with voriconazole. Antimicrob Agents Chemother. 2006;50:96-103.

17. Menezes EA, Vasconcelos Júnior AA, Silva CL, Plutarco FX, Cunha Mda C, Cunha FA. In vitro synergism of simvastatin and fluconazole against Candida species. Rev Inst Med Trop Sao Paulo. 2012;54:197-199.

18. Nyilasi I, Kocsubé S, Krizsán K, Galgóczy L, Papp T, Pesti M, Nagy K, Vágvölgyi C. Susceptibility of clinically important dermatophytes against statins and different statinantifungal combinations. Sabouraudia. 2013;52:140-148.

19. Nyilasi I, Kocsubé S, Krizsán K, Galgóczy L, Pesti M, Papp $\mathrm{T}$, et al. In vitro synergistic interactions of the effects of various statins and azoles against some clinically important fungi. FEMS Microbiol Lett. 2010;307:175-184.

20. Brilhante RS, Caetano EP, Oliveira JS, Castelo-Branco DD, Souza ER, Alencar LP, Cordeiro RD, Bandeira TD, Sidrim JJ, Rocha MF. Simvastatin inhibits planktonic cells and biofilms of Candida and Cryptococcusspecies. Brazilian Journal of Infectious Diseases. 2015;19:459-465.

21. Lima WG, Alves-Nascimento LA, Andrade JT, Vieira L, de Azambuja Ribeiro RIM, Thomé RG, et al. Are the Statins promising antifungal agents against invasive candidiasis?. Biomed Pharmacother. 2019;111:270-281.

22. Parihar SP, Guler R, Brombacher F. Statins: A viable candidate for host-directed therapy against infectious diseases. Nat Rev Immunol. 2019;19:104-117.

23. Zhou Y, Yang H, Zhou X, Luo H, Tang F, Yang J, et al. Lovastatin synergizes with itraconazole against planktonic cells and biofilms of Candida albicans through the regulation on ergosterol biosynthesis pathway. Appl Microbiol Biotechnol. 2018;102:5255-5264.

24. Ting M, Whitaker EJ, Albandar JM. Systematic review of the in vitro effects of statins on oral and perioral microorganisms. Eur J Oral Sci. 2016;124:4-10.

25. Maheronnaghsh M, Tolouei S, Dehghan P, Chadeganipour M, Yazdi M. Identification of Candida species in patients with oral lesion undergoing chemotherapy along with minimum inhibitory concentration to fluconazole. Adv Biomed Res. 2016;5:132.

26. Wayne P. Clinical and Laboratory Standards Institute: Reference method for broth dilution antifungal susceptibility testing of yeasts; approved standard. CLSI document M27-A3 and Supplement S. April 2008.

27. Powderly WG, Kobayashi GS, Herzig GP, Medoff G. Amphotericin B-resistant yeast infection in severely immunocompromised patients. Am J Med. 1988;84:826-832.

28. Dick JD, Merz WG, Saral R. Incidence of polyene-resistant yeasts recovered from clinical specimens. Antimicrob Agents Chemother. 1980;18:158-163.

29. Farmakiotis D, Tarrand JJ, Kontoyiannis DP. Drug-resistant Candida glabrata infection in cancer patients. Emerg Infect Dis. 2014;20:1833-1840.

30. Bagg J, Sweeney MP, Lewis MA, Jackson MS, Coleman D, Al MA, et al. High prevalence of non-albicans yeasts and detection of anti-fungal resistance in the oral flora of patients with advanced cancer. Palliat Med. 2003;17:477-481. 
31. Anaissie EJ, McGinnis MR, Pfaller MA. Clinical Mycology, 1st ed. New York: Churchill Livingstone; 2003: pp. 162-172.

32. Nicolatou-Galitis O, Sotiropoulou-Lontou A, Velegraki A, Pissakas G, Kolitsi G, Kyprianou K, et al. Oral candidiasis in head and neck cancer patients receiving radiotherapy with amifostine cytoprotection. Oral Oncol. 2003;39:397-401.

33. Karbach J, Walter C, Al-Nawas B. Evaluation of saliva flow rates, Candida colonization and susceptibility of Candida strains after head and neck radiation. Clin Oral Investig, 2012;16:1305-1312.

34. Wilson D, Thewes S, Zakikhany K, Fradin C, Albrecht A, Almeida $\mathrm{R}$, et al. Identifying infection-associated genes of
Candida albicans in the postgenomic era. FEMS Yeast Res. 2009;9:688-700.

35. Brown AJ, Odds FC, Gow NA. Infection-related gene expression in Candida albicans. Curr Opin Microbiol. 2007; 10:307-313.

36. Jones PH, Davidson MH, Stein EA, Bays HE, McKenney JM, Miller E, et al. Comparison of the efficacy and safety of rosuvastatin versus atorvastatin, simvastatin, and pravastatin across doses (STELLAR* Trial). Am J Cardiol. 2003;92:152-160.

37. Qiao J, Kontoyiannis DP, Wan Z, Li R, Liu W. Antifungal activity of statins against Aspergillus species. Med Mycol. 2007;45:589-593 